\title{
68yr Old Female with Post Menopausal Bleeding
}

\author{
Sushila B. Ladumor ${ }^{1 *}$ Adham Darweesh $^{2}$ \\ ${ }^{1}$ Consultant Radiologist, Clinical Imaging Department, Hamad Medical Corporation, Qatar \\ ${ }^{2}$ Senior Consultant Radiologist, Clinical Imaging Department, Hamad Medical Corporation, Qatar
}

Submission: November 11, 2017; Published: December 08, 2017

*Corresponding author: Sushila Ladumor B, Consultant Radiologist, Clinical Imaging Department, Hamad Medical Corporation, Assistant Professor in Clinical Radiology, Weil Cornel Medical College, Doha, Qatar, Email: drsbladumor@yahoo.com

\section{Abstract (Uterine adenomyosis)}

With the help of recent advanced imaging plays amazing role in non-invasive diagnosis of uterine adenomyosis, usually common condition characterized by the presence of heterotopic non-malignant endometrial glands and stroma in the myometrium with hyperplasia of the adjacent smooth muscle [1].

Uterine adenomyosis is a condition in which the inner lining of the uterus (the endometrium) breaks through the muscle wall of the uterus (the myometrium). Patient can be asymptomatic or it may cause atypical pain and chronic vaginal bleeding which does not respond to usual hormonal therapy or uterine evacuation. The other common symptoms apart from pelvic pain and dysmenorrhea, menstrual cramps, lower abdominal heaviness and bloating before menstrual periods. However, these symptoms are nonspecific and can be associated with other pathology such as dysfunctional uterine bleeding, leiomyoma, and endometriosis [1].

Clinical History: Presenting case of uterine adenomysis and ovarian thecoma-fibroma in a 68year old lady presenting with post-menopausal bleeding. No previous Imaging or any prior consultation in system.

\section{Ovarian thecoma-fibroma}

Ovarian thecoma-fibroma groups (OTFG) are uncommon sex cord-stromal neoplasms (mesenchymal origin). They are thought to account for approximately $0.5-1 \%$ of all ovarian tumors. It is described as functional ovarian tumors as they secrete estrogen. Fibromas and fibrothecomas greater than $6 \mathrm{~cm}$ diameter are more likely to have presence of capsule, peripheral cystic degenerative changes along with heterogeneous T1, T2 signal and heterogeneous enhancement [2].

Keywords: Adenomyosis; Junctional zone; Ectopic endometriosis; Endometrial cyst; Fibrothecoma; Imaging features; MRI; Ovarian fibroma; Ovarian neoplasms; Ultrasound; Pathology

\section{Imaging Work-Up}

Patient referred to pelvic US and US showed uterine adenomyosis and right adnexal mass (Images are not gown). Patient referred for MRI for better evaluation and characterization of uterine and ovarian lesion.

There is a large complex right adnexal lesion stretching the broad ligament at anterosuperior aspect measures approximately $10.5 \times 7.7 \times 12.9 \mathrm{~cm}$ in maximum AP, transverse and craniocaudal diameter shows iso and low signal in $\mathrm{T} 2$ shown by star in Figure 1a. Small cyst at on right aspect of uterus and superior aspect of right adnexal mass representing ovarian follicles. Both ovaries are not seen separately. Another elongated cystic area at left site of lesion demonstrates non-enhancing thin septa which measures approximately $8.9 \times 3 \mathrm{~cm}$ in maximum AP and transverse diameter. This lesion is stretching the cervix and displaced the uterus to the left side Figure 2,3.

\section{Impression of MRI Pelvis}

Enlarge retroflex uterus demonstrated evidence of diffuse adenomyosis more involvement of the right anterior fundal region. Multiple myometrial and sub serosal fibroids. The right adnexal mass with focal area appears abutting the right lateral wall of the upper cervical segment most likely ovarian origin of fibrous etiology (fibroma-thecoma) or other less likely possibility of pedunculated fibroid cannot be excluded.

Laboratory Findings:

CA $125={ }^{*} \mathrm{H} 79 \mathrm{U} / \mathrm{mL}(\mathrm{HI})$

CA $15-321 \mathrm{U} / \mathrm{mL}$

CA $19-9=* H 44 \mathrm{U} / \mathrm{mL}(\mathrm{HI})$

$\mathrm{CEA}=* 1.0 \mathrm{Ug} / \mathrm{L}$

Treatment: Patient underwent surgery. 


\section{Open Access Journal of Surgery}

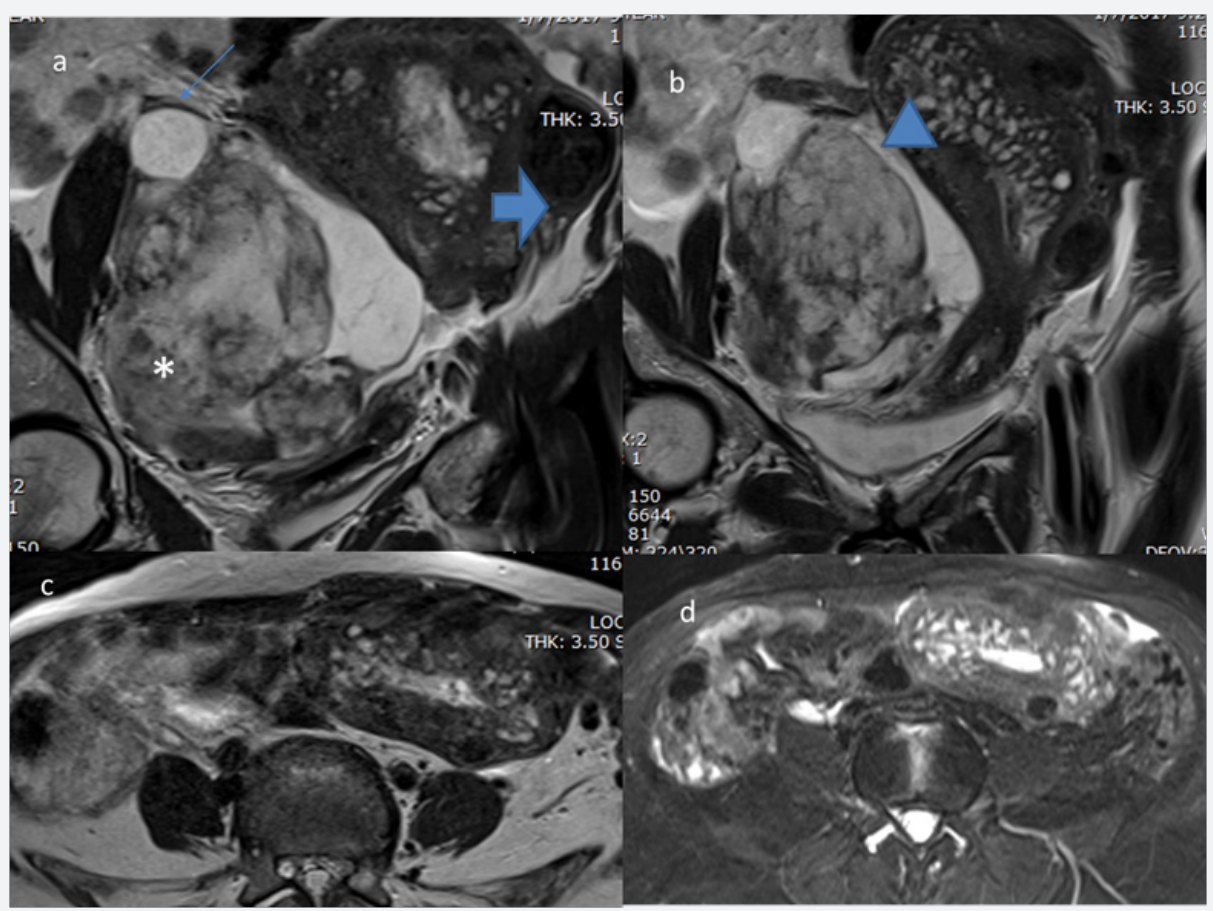

Figure 1: T2 TSE coronal (a\&b), T2 axial (c) and T2 fat sat axial (d) Uterus is mildly retroflexed and is enlarged. Diffuse widening of junctional zone demonstrates multiple tiny cysts of variable size with more involvement of the right anterior fundal region with maximum thickness measures about $33 \mathrm{~mm}$ Arrow head in Figure b). The endometrial cavity demonstrates irregular in outline with maximum thickness in fundus of uterus measures about $6.5 \mathrm{~mm}$. Multiple small fibroids of variable size in myometrial and sub serosal location with the largest one noted at the left anterior fundal region (shown by thick arrow in Figure a).

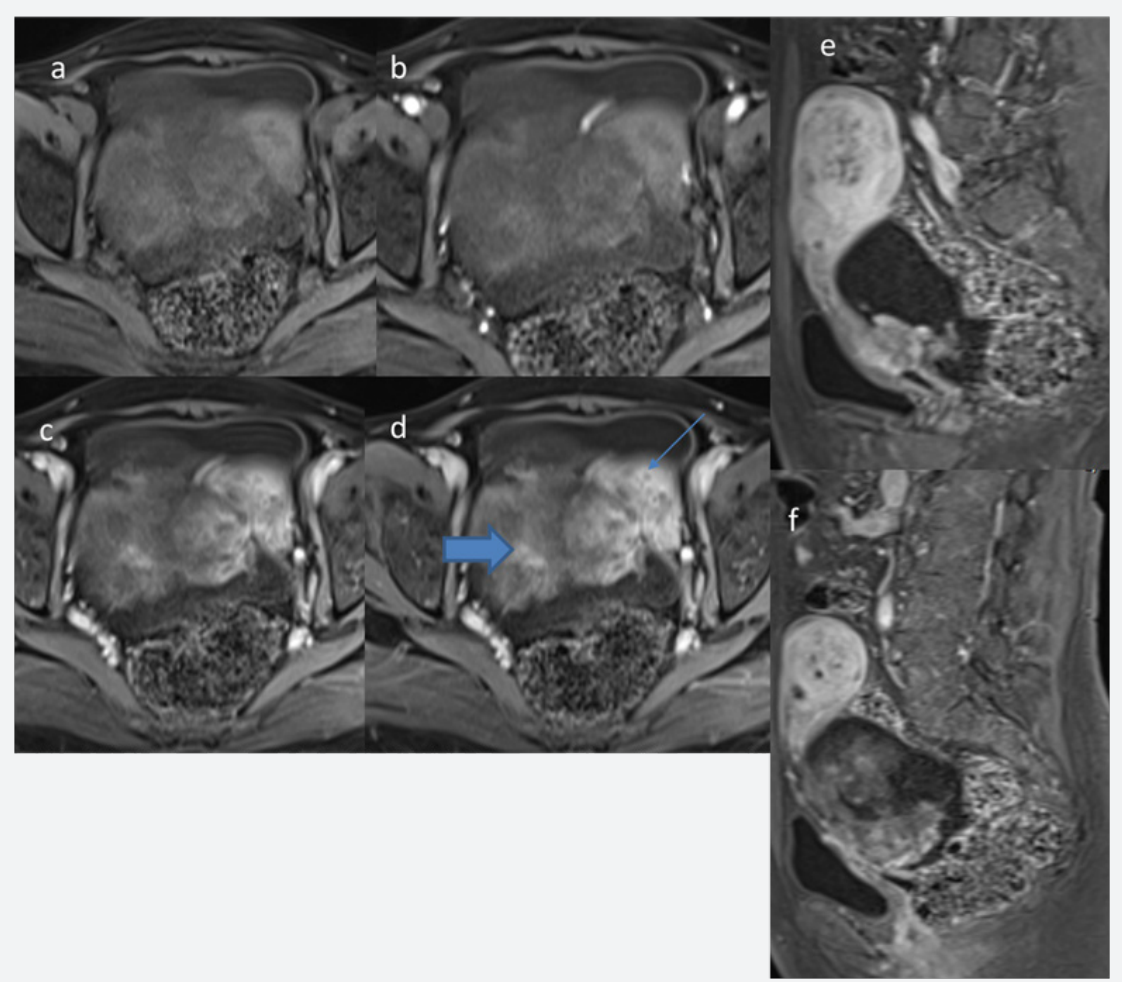

Figure 2: Pre-contrast T1 axial (a) and post-contrast dynamic axial (b,c,d,) sagittal (e,f). Right adnexal mass showing less enhancement (thick arrow in Figure d) compared to uterine myometrium (thin arrow in Figure d) and uterine fibroid. No definite restricted diffusion and intralesional fat (images of diffusion and in-phase-opposed phase not shown). No hemorrhagic component. 


\section{Histopathology}

\section{Diagnosis}

A. Uterus, cervix, right and left fallopian tubes and ovaries, total hysterectomy with bilateral salpingo-oophorectomy:

a) Endometrium: Simple hyperplasia with benign endometrial polyp

b) Myometrium: - Intramural leiomyomata, the largest measures $2.5 \mathrm{~cm}$

c) Extensive adenomyosis.

d) Cervix: - squamous metaplasia

e) Right and left fallopian tubes: No specific histopathologic findings.

f) Right ovary: No specific histopathologic findings

g) Left ovary: Thecoma / fibroma, $10 \mathrm{~cm}$

B. Omentum, omentectomy:

a) Adipose tissue with no specific histopathologic findings.

\section{Discussion (Uterine adenomyosis)}

Adenomyosis was first described by Rokitansky in 1860 and its endometriotic origin was highlighted by Thomas Stephen Cullen in 1921. Uterine adenomyosis is relatively common, non-malignant pathology. It is thought by many to be on the spectrum of endometriosis, with ectopic endometrial tissue in the myometrium. High resolution Ultrasound is first choice of imaging modality and in some cases MRI is second choice for accurate diagnosis and to determine the extent and involvement of myometrial penetration.

Hysterectomy is the definitive treatment for debilitating adenomyosis and uterus-conserving therapy/myomectomy is possible in cases of leiomyoma. Accurate Imaging diagnosis of myometrial involvement is very important as it help in planning of definite treatment because superficial adenomyosis demonstrates significant responds to endometrial ablation than deep adenomyosis. Also imaging is helpful for followup in monitoring response of the disease in patients receiving conservative therapy. Easy availability of high-resolution imaging techniques, adenomyosis can be diagnosed with a high degree of confidence. The imaging findings easily seen with endovaginal ultrasonography (US) and magnetic resonance (MR) imaging.

\section{Epidemiology}

Adenomyosis typically affects women of reproductive age. In general, affected women who are multiparous and the condition are seen more in a woman with a history of previous uterine intervention procedure (e.g. Caesarian section, dilatation and curettage). The reported incidence ranges widely from 5 to
$70 \%$, depending on the histological definition or the imaging modality used [3].

\section{Clinical presentation}

Majority of patients with adenomyosis are symptomatic and dominant complaint of menorrhagia and dysmenorrhea or may present with chronic pelvic pain or menstrual menometrorrhagia. Heterotopic endometrial glands within the myometrium do not respond to cyclic ovarian hormones, while endometriosis usually responds with cyclic ovarian hormones.

\section{Associations}

Co-existent endometriosis reported in $20 \%$ of cases. Radiographic features [2,3] Imaging features are variable and in many instances very subtle. Three (some say four) forms can be distinguished: Adenomyosis is usually relatively generalized, affecting large portions of the uterus (typically the posterior wall), but sparing the cervix. Even with diffuse enlargement of the uterus, the overall contour is usually preserved [4].

In some cases, adenomyosis may be localized, forming a mass. In such cases, the term adenomyoma may be used, although there appears to be some disagreement about whether the terms focal adenomyosis and adenomyoma refer to exactly the same entity. In these cases sometime it is seen as focal mass by US same as uterine fibroid while MRI is more helpful to characterize the lesion as it shows diffuse focal thickening of junction zone with tiny $\mathrm{T} 2$ high signal cyst and sometime T1 high signal tiny areas.

\section{Ultrasound}

Pelvic ultrasound is usually the first and often the only imaging modality to work-up in patient with history of menorrhagia and dysmenorrhea. Unfortunately, the sonographic features of adenomyosis are variable and may be absent. The reported sensitivity and specificity of trans-abdominal ultrasound are 32$63 \%$ and 95-97\% respectively [3].

\section{The spectrum of findings includes}

Transvaginal US has more sensitivity than transabdominal US with findings are variable. In minimal or early cases the uterus appears within normal limit. Can be seen as focal or diffuse bulky myometrium, more of posterior wall seen as thickening of transitional zone (sometimes seen as a hypo echoic halo surrounding the endometrial layer of more than $12 \mathrm{~mm}$ thickness). It can be seen as sub endometrial echogenic linear striations or hyperechoiec tiny nodules or small myometrial/ sub endometrial cysts. Globular uterine enlargement with heterogenous myometrial echo texture without evidence of uterine fibroid can be seen in some cases. Loss of clear definition of endometrial/myometrial border due to invasion of the myometrium is another imaging feature. When an adenomyoma is present, appearances may closely mimic those of a uterine fibroid, which may also co-exist as in this case. 
MRI

Pelvic MRI is the modality of choice to diagnose and characterize adenomyosis with high degree of confidence, and T2 weighted axial and sagittal images are significantly useful. MRI has a sensitivity of 78-88 \% and a specificity of $67-93 \%$ [3]. Widening of the junctional zone of the uterus to more than $12 \mathrm{~mm}$ is most evident finding and easily recognized. Normal junctional zone thickness is up to $5-8 \mathrm{~mm}$ ). T2 weighted sagittal and axial sequences are most useful images reveal diffuse or illdefined focal junctional zone thickening with very small high signal cystic changes or may have high signal striations.

Diffuse globular enlargement can be seen easily. T1 weighted sequences often reveals foci of high signal intensity suggesting menstrual hemorrhage into heterotopic endometrial tissue. Diffusion weighted images usually not indicated but also demonstrates diffuse or ill-defined focal junctional zone thickening with very small high signal cystic changes or may have high signal striations with no diffusion restriction in high b value. T1 weighted post-contrast enhanced evaluation is usually not indicated for evaluation of adenomyosis, however, if performed, it shows enhancement of the ectopic endometrial glands.

\section{Treatment and Prognosis}

Treatment depends on the severity of symptoms and the need to preserve fertility. In some instances, suppression of normal cyclical hormone-induced proliferation of endometrial tissue (e.g. GnRH agonist) is sufficient. In women with severe symptoms not relieved medically and fertility is not a problem or concern than definitive treatment as hysterectomy may be performed.

\section{Differential diagnosis}

The differential depends on the macroscopic distribution of endometrial tissue.

\section{Conclusions}

With the help of modern high-resolution imaging techniques such as endovaginal US and MRI, adenomyosis can be diagnosed with a high degree of confidence with many imaging findings as described above. The imaging signs demonstrated with endovaginal US and MR imaging correspond closely to the varied appearances of this disease at histopathologic analysis. Radiologist, Sonologists and sonographers should familiarize themselves with the endovaginal US appearance of this disease. Endovaginal US can be used as the initial imaging modality in patients suspected of having adenomyosis, but US must be performed meticulously and in real time. MR imaging can be reserved for cases that are indeterminate at endovaginal US and for patients who will undergo uterus-sparing surgery. Endovaginal US is modality of choice in follow-up cases.

\section{Discussion (Ovarian thecoma-fibroma)}

Ovarian thecoma-fibroma groups (OTFG) neoplasms usually occur in postmenopausal women (more than $80 \%$ ), often with a good prognosis. Complaint of abdominal pain and abdominal bulge in cases with large tumors. The coma is benign lesion and occasionally malignant, which may secrete estrogen and lead to symptoms such as menstrual disorders and postmenopausal bleeding and more than $20 \%$ have endometrial carcinoma [5-7]. Large tumors were often associated with torsion, hemorrhage, calcification, or complicated with other cystic lesions, which showed mixed echogenic masses. Sixty percent of postmenopausal patients present with uterine bleeding, and more than $20 \%$ have endometrial carcinoma.

Ovarian fibromas and fibrothecomas are benign neoplasms that can confuse with malignant ovarian masses due to solid adnexal masses, sometimes associated with as cites and pleural effusions. Sonography is usually used as the first-line imaging technique for the evaluation of ovarian pathologic abnormalities. However, ultrasound features of fibromas and fibrothecomas are usually not typical [5], and MRI is often required for further characterization of ovarian fibromas and fibrothecomas from other solid ovarian tumors particularly from pedunculated or broad ligament leiomyomas. A finding of normal ovaries was initially thought to exclude ovarian fibromas and fibrothecomas [5], a report that has been contradicted by a more recent study in which the ipsilateral ovary was identified in $46 \%$ of cases [5].

MRI is an excellent imaging modality for the detection and differentiation of ovarian fibromas and fibrothecomas. It usually demonstrates homogeneous iso to low signal intensity on T1weighted images and predominantly iso to low signal intensity on T2-weighted images compare to uterine myometrium and iliopsoas muscle. No intra-lesional fatty component or calcification (in contrast to ovarian dermoid/teratoma). Usually no diffusion restriction. Post-contrast sequences reveal significant less enhancement of fibromas and fibrothecomas about $75 \%$ than uterine myometrium and fibroids. Proper diagnosis of these tumors on imaging can help to reduce patient tension and relieve from unnecessary invasive procedures.

MRI features of fibromas and fibrothecomas depend on the size of the lesion. The presence of pseudo capsule, degenerative changes, peripheral sub capsular cystic areas, heterogeneous T2 signal, and heterogeneous enhancement are more common with larger fibromas and fibrothecomas, and at least four or more of these five features were present in $93 \%$ of fibromas and fibrothecomas larger than $6 \mathrm{~cm}$. Peripheral small cysts can be seen due to stretched ovarian stroma around the fibromas and fibrothecomas, and it usually confirm ovarian origin of the lesion, thus helping in differentiation from fibroids [6-9]. 


\section{Conclusion}

MRI features of ovarian fibromas and fibrothecomas depend on size. Degenerative changes common with fibromas and fibrothecomas larger than $6 \mathrm{~cm}$. Fibromas and fibrothecomas enhance less compare to myometrium and fibroids, and less than $75 \%$ maximum percentage enhancement can help in differentiating fibromas and fibrothecomas from fibroids. The typical sonographic features of OTFG include adnexal hypo echoic masses with clear border and acoustic attenuation as well as minimal Doppler flow signals. All imaging features improve the preoperative diagnostic accuracy $[1,8,9]$.

\section{References}

1. Reinhold C, Tafazoli F, Mehio A, Wang L, Atri M, et al. (1999) Uterine Adenomyosis: Endovaginal US and MR Imaging Features with Histopathologic Correlation. RSNA 19(1).

2. Shinagare AB, Meylaerts LJ, Laury AR, Mortele KJ, (2012) MRI Features of Ovarian Fibroma and Fibrothecoma with Histopathologic Correlation. AJR Am J Roentgenol 198(3): 296-303.
3. Tamai K, Togashi K, Ito T, Morisawa N, Fujiwara T, et al. (2005) MR Imaging Findings of Adenomyosis: Correlation with Histopathologic Features and Diagnostic Pitfalls. RSNA 25(1).

4. Hulka CA, Hall DA, Mc Carthy K, Simeone J (2002) Sonographic Findings in Patients with Adenomyosis: Can Sonography Assist in Predicting Extent of Disease. AJR 179(2): 379-383.

5. Matthew Lukies, Frank Gaillard. Adenomyosis of the uterus.

6. Weinreb JC, Barkoff ND, Megibow A, Demopoulos R (1990) The value of MR imaging in distinguishing leiomyomas from other solid pelvic masses when sonography is indeterminate. AJR Am J Roentgenol 154(2): 295-299.

7. Hui Chen, Yan Liu, Li-fei Shen, Mei-jiao Jiang, Zhi-fang Yang, et al. (2016) Ovarian thecoma-fibroma groups: clinical and sonographic features with pathological comparison. Journal of Ovarian Research 9: 81.

8. Tanaka YO, Tsunoda H, Kitagawa Y, Ueno T, Yoshikawa H, et al. (2004) Functioning Ovarian Tumors: Direct and Indirect Findings at MR Imaging. Radiographics 24(1): 147-166.

9. Radswiki Ovarian fibroma.

\section{Your next submission with Juniper Publishers will reach you the below assets}

- Quality Editorial service

- Swift Peer Review

- Reprints availability

- E-prints Service

- Manuscript Podcast for convenient understanding

- Global attainment for your research

- Manuscript accessibility in different formats

( Pdf, E-pub, Full Text, Audio)

- Unceasing customer service

Track the below URL for one-step submission https://juniperpublishers.com/online-submission.php 